\title{
Características das Partes Não-Integrantes da Carcaça de Novilhos 5/8Nelore 3/8Charolês Abatidos em Três Estádios de Desenvolvimento
}

\section{João Restle ${ }^{1}$, Luís Fernando Glasenapp de Menezes ${ }^{2}$, Miguelangelo Ziegler Arboitte ${ }^{3}$, Leonir Luiz Pascoal ${ }^{4}$, Paulo Santana Pacheco ${ }^{5}$, João Teodoro Pádua ${ }^{6}$}

\begin{abstract}
RESUMO - Foram avaliadas as características das partes não-integrantes da carcaça de novilhos 5/8 Nelore 3/8 Charolês terminados em confinamento e abatidos com 425, 467 e $510 \mathrm{~kg}$. Os períodos de alimentação foram 30, 65 e 94 dias, respectivamente. A idade e peso médio iniciais foram de 660 dias e $361 \mathrm{~kg}$, respectivamente. A dieta alimentar, com relação volumoso:concentrado de 60:40 com base na matéria seca (MS), continha 10,25\% de proteína bruta e 3,18 Mcal de energia digestível/kg MS. De acordo com estimativa da equação de regressão, os rendimentos de carcaças quente e fria elevaram com o aumento no peso de abate (P). No entanto, quando expressos por peso de corpo vazio (PCV), houve similaridade entre os diferentes tratamentos. Quando os componentes foram expressos em valores absolutos, couro, total de componentes externos (TCE), fígado, total de órgãos vitais (TOV), total de gorduras internas (TGORD) e total de trato gastrintestinal vazio (TGVZ) apresentaram incremento com o aumento no P. Quando expressos por $100 \mathrm{~kg}$ de P e PCV, os componentes couro, TCE, fígado e TGVZ apresentaram similaridade; coração e TOV, decréscimo; e TGORD, acréscimo, com o aumento no P. Houve correlação positiva entre peso de fígado com consumo de matéria seca $(\mathrm{r}=0,91)$ e com energia líquida de mantença (ELm) $(\mathrm{r}=0,91)$ e entre TGORD com ELm $(\mathrm{r}=0,78)$.
\end{abstract}

Palavras-chave: consumo de matéria seca, fígado, gordura, mestiços, peso de abate, peso de corpo vazio

\section{Characteristics of Non Integrant Parts of the Carcass of 5/8 Nellore 3/8 Charolais Steers Slaughtered at Three Maturity Stages}

\begin{abstract}
The characteristics of the body parts non-integrant of the carcass of 5/8 Nellore 3/8 Charolais steers feedlot finished and slaughtered with 425, 467 and $510 \mathrm{~kg}$, were evaluated. The feeding periods were 30,65 and 94 days, respectively. The average initial age and weight were 660 days and $361 \mathrm{~kg}$, respectively. The diet, with $60 \%$ roughage and $40 \%$ concentrate dry matter basis (DM), contained $10.25 \%$ of crude protein and $3.18 \mathrm{Mcal}$ of digestible energy $/ \mathrm{kg} \mathrm{DM}$. According to regression equation estimate, the hot and cold carcass dressing increased as slaughter weight (SW) increased. However, when expressed per empty body weight (EBW), similarity among the different treatments was verified. When the components were expressed in absolute values, rawhide, total external components (TEC), liver, total vital organs (TVO), total internal fats (TFAT) and total empty gastrintestinal tract (TEGT) showed increase with increase in SW. When expressed per $100 \mathrm{~kg}$ of body weight and EBW, the rawhide, TEC, liver and TEGT components showed similarity; heart and TVO showed decrease; and TFAT showed increase, with increase in SW. Positive correlations between liver weight with dry matter intake $(\mathrm{r}=.91)$ and with net energy maintenance $(\mathrm{NEm})(\mathrm{r}=.91)$, and between TFAT with NEm $(\mathrm{r}=.78)$ were observed.
\end{abstract}

Key Words: crossbreds, dry matter intake, empty body weight, fat, liver, slaughter weight

\section{Introdução}

Importante alternativa para o avanço na produção de carne bovina e a remuneração por animal abatido seria o aumento do peso de abate dos animais. Isto porque o peso de abate, os pesos e os rendimentos de carcaça quente e/ou fria são as principais características envolvidas no processo de comercialização entre frigorífico e produtor (Restle et al., 1997a; Costa et al., 2002a; Arboitte et al., 2004a).
No entanto, cada vez mais os componentes nãointegrantes tornam-se importante fonte de receita para os frigoríficos, que os comercializam tanto no mercado interno quanto no externo. Apesar de o produtor não receber remuneração por estes componentes, estes são importantes, pois afetam o rendimento de carcaça e as exigências nutricionais, principalmente a de energia líquida de mantença (Galvão et al., 1991; Jorge et al., 1999; Véras et al., 2001; Pacheco et al., 2005a). Com isso, em bovinos de

\footnotetext{
${ }^{1}$ Engenheiro Agrônomo, PhD. Pesquisador Visitante/CNPq - DPA/UFG. E.mail: jorestle@terra.com.br

2 Zootecnista, MSc. Doutorando do PPGZ da UFSM. E.mail: Ifgdm@pop.com.br

3 Zootecnista, MSc. Professor substituto do Departamento de Zootecnia da UFSM.

4 Zootecnista, MSc. Professor Assistente do Departamento de Zootecnia da UFSM.

5 Zootecnista, MSc. Doutorando PPGCA - UFG.

6 Zootecnista, Dr., Professor Adjunto do Departamento de Produçào Animal - EV/UFG.
} 
corte, a avaliação dos componentes do corpo vazio deve ser utilizada como informação auxiliar no entendimento de características relacionadas ao desempenho e à carcaça dos animais (Pacheco et al., 2005a).

Notadamente, o couro, além dos órgãos internos, representa uma parcela significativa da receita dos frigoríficos. O couro é, sem dúvida, o componente de maior importância, se considerado o valor agregado que recebe do abate até a transformação em produtos comerciais. O peso do couro é influenciado pelo peso de abate e pelo grupo genético dos animais (Galvão et al., 1991; Signoretti et al., 1999; Pacheco et al., 2005a), podendo ocorrer decréscimo (Galvão et al., 1991) ou acréscimo em seu peso (Signoretti et al., 1999; Jorge \& Fontes, 2001) quando expresso em relação ao peso de corpo vazio, com o aumento no peso de abate dos animais.

Em relação aos demais componentes do corpo vazio, o aumento no peso de abate dos animais pode resultar em diferentes comportamentos. Nos estudos de Oliveira et al. (1992) e Signoretti et al. (1999), houve decréscimo no peso relativo dos órgãos internos relativos no peso de corpo vazio com o aumento do peso dos animais. Similaridade no peso dos órgãos vitais ajustado para peso de corpo vazio de novilhos abatidos em diferentes estádios de maturidade (405, 450 e $500 \mathrm{~kg}$ ) foi relatada por Jorge et al. (1999), enquanto Galvão et al. (1991) verificaram redução no peso de pés e cabeça/100 $\mathrm{kg}$ de corpo vazio com o avanço da maturidade dos animais de 90 para $110 \%$ do peso adulto.

Este trabalho foi realizado objetivando-se avaliar os componentes não-integrantes da carcaça de novilhos 5/8 Nelore 3/8 Charolês terminados em confinamento, abatidos em três estádios de maturidade.

\section{Material e Métodos}

O experimento foi realizado no Setor de Bovinocultura de Corte do Departamento de Zootecnia da Universidade Federal de Santa Maria (UFSM), situada na Depressão Central do estado do Rio Grande do Sul, com coordenadas $29^{\circ} 42^{\prime}$ de latitude Sul e $54^{\circ} 42^{\prime}$ de longitude Oeste e 96 metros de altitude.

Foram utilizados 18 novilhos do grupo genético 5/8Nelore 3/8Charolês, contemporâneos e provenientes do mesmo rebanho, com idade, peso e estado corporal médio iniciais de 660 dias, $361 \mathrm{~kg}$ e 2,91 pontos (escala de 1 a 5 segundo Restle, 1972), respec- tivamente. Os animais foram distribuídos em três tratamentos, que corresponderam aos pesos de abate pretendidos de 420, 460 e $500 \mathrm{~kg}$, obtendo-se pesos reais de 425,467 e $510 \mathrm{~kg}$. Os períodos de alimentação foram 30, 65 e 94 dias, respectivamente.

Os animais, alimentados duas vezes ao dia, receberam a metade da dieta pela manhã $(8 \mathrm{~h})$ e o restante à tarde $(16 \mathrm{~h})$. Diariamente, pela manhã, eram retiradas e pesadas as sobras de alimento do dia anterior, para ajuste da quantidade de alimento ofertado e posterior cálculo do consumo. A quantidade de matéria seca (MS) fornecida diariamente foi $10 \%$ superior ao consumo voluntário observado. A dieta foi calculada segundo o NRC (1996), objetivando ganho de peso médio de $1,600 \mathrm{~kg} /$ animal/dia.

Na Tabela 1, encontra-se a composição percentual dos componentes da dieta experimental, expressos em MS, utilizando-se relação volumoso:concentrado de 60:40. O volumoso foi representado por silagem de milho de alta qualidade $(46,5 \%$ de grãos na massa ensilada), enquanto a dieta apresentou em média $10,25 \%$ de proteína bruta e 3,18 Mcal de energia digestível/kg de MS.

Conforme os animais dos tratamentos atingiram o peso pré-determinado, foram pesados (peso de abate) após jejum de sólidos de 14 horas, transportados ao frigorífico comercial e abatidos segundo o fluxo normal do estabelecimento.

Tabela 1 - Composição da dieta, com base na matéria seca

Table 1 - Diet composition, dry matter basis

\begin{tabular}{lc}
\hline $\begin{array}{l}\text { Ingrediente } \\
\text { Ingredient }\end{array}$ & $\begin{array}{c}\text { Composição } \\
\text { Composition }\end{array}$ \\
\hline $\begin{array}{l}\text { Silagem de milho, \% } \\
\text { Corn silage, \% }\end{array}$ & 60,00 \\
$\begin{array}{l}\text { Farelo de trigo, \% } \\
\text { Wheat bran, \% }\end{array}$ & 28,24 \\
$\begin{array}{l}\text { Sorgo em grão moído, \% } \\
\text { Ground sorghum grain, \% }\end{array}$ & 10,07 \\
$\begin{array}{l}\text { Uréia (45-00-00), \% } \\
\text { Urea (45-00-00), \% }\end{array}$ & 0,17 \\
Calcário calcítico, \% & \\
$\begin{array}{l}\text { Limestone, \% } \\
\text { Cloreto de sódio (NaCl), \% }\end{array}$ & 1,02 \\
$\begin{array}{l}\text { Sodium chloride (NaCl), \% } \\
\text { Monensina sódica, \% } \\
\text { Sodium monensin, \% }\end{array}$ & 0,48 \\
\end{tabular}


No momento do abate, foram coletados os pesos dos seguintes componentes: sangue; cabeça (incluindo orelhas e chifres), vassoura da cauda, patas e couro (denominados componentes externos); coração, rins, fígado, baço e pulmões (denominados órgãos vitais); rúmen-retículo, omaso, abomaso e intestinos delgado e grosso vazios (denominados trato gastrintestinal vazio); gorduras inguinal, renal, ruminal e de toalete (denominadas gorduras internas).

As meia-carcaças (direita e esquerda) foram identificadas, sendo o peso de carcaça quente obtido anteriormente à entrada das mesmas na câmara de resfriamento.

O peso de corpo vazio foi obtido pelo somatório dos pesos de carcaça quente, sangue, componentes externos, órgãos vitais, trato gastrintestinal vazio e gorduras internas.

Após 24 horas de resfriamento em câmara fria a $2^{\circ} \mathrm{C}$, as carcaças foram novamente pesadas, obtendose o peso de carcaça fria. A partir dos pesos de abate, de corpo vazio, de carcaça quente e de carcaça fria, calculou-se os rendimentos de carcaça quente e fria em relação ao peso de abate e de corpo vazio.

O delineamento experimental utilizado foi o inteiramente casualizado, com três tratamentos e seis repetições, considerando cada animal uma unidade experimental.

Os dados e características de desempenho dos animais, apresentadas por Arboitte et al. (2004 b), foram submetidos às análises de regressão e correlação de Pearson por intermédio do programa estatístico SAS (1997).

O modelo matemático utilizado foi:

$$
\mathrm{Y}_{i j l m}=\mathrm{B}_{0}+\mathrm{Pi}_{i}+\mathrm{B}_{1} \mathrm{P}_{j l}+\mathrm{E}_{i j l m},
$$

em que: $\mathrm{Y}_{i j l m}=$ variáveis dependentes; $\mathrm{B}_{0}$; $\mathrm{B}_{1}=$ parâmetros da equação a serem estimados; $\mathrm{Pi}_{i}=$ efeito do ${ }_{i}$-ésimo peso inicial dos novilhos, utilizado como covariável; $\mathrm{P}_{j l}=$ peso da abate na ${ }^{-}$ ésima repetição do ${ }_{j}$-ésimo tratamento; $\mathrm{E}_{i j l m}=$ erro experimental aleatório, $\operatorname{NID}\left(0, \sigma^{2}\right)$.

Os dados foram testados quanto à normalidade, pelo teste de Shapiro-Wilk (SAS, 1997), sendo efetuada quando necessário, a transformação da raiz quadrada dos dados das variáveis.

\section{Resultados e Discussão}

Na Tabela 2, constam os resultados referentes a algumas características de desempenho dos animais deste estudo, segundo Arboitte et al. (2004b).

Os valores médios referentes ao peso de corpo vazio $(\mathrm{PCV})$, rendimentos de carcaça quente e fria expressos por peso de abate $(\mathrm{P})$ e por peso de corpo vazio encontram-se na Tabela 3.

Verifica-se que os rendimentos de carcaça quente e fria expressos por $100 \mathrm{~kg}$ de peso de abate elevaram de 0,026 e $0,028 \%$, respectivamente, para cada $\mathrm{kg}$ de aumento no peso de abate, segundo estimativas das equações de regressão. No entanto, quando expressos por peso de corpo vazio, houve similaridade entre os diferentes tratamentos, com média de 63,04 e $61,67 \%$, respectivamente.

$\mathrm{Na}$ Tabela 4, constam os valores médios referentes ao peso absoluto e relativo ao P e PCV dos diferentes componentes externos, de acordo com o peso de abate.

Houve resposta linear nos pesos absolutos de patas e couro com o aumento no peso de abate, com acréscimo mais proeminente neste último componente, elevando $22,5 \%$ dos 425 aos $467 \mathrm{~kg}$ e $3,8 \%$ dos 467 aos $510 \mathrm{~kg}$. Para o frigorífico, o resultado verificado para o peso absoluto de couro é importante, uma vez que representa maior receita por unidade comercializada. Avaliando os componentes não-integrantes da carcaça de novilhos jovens e superjovens dos grupos genéticos 5/8 Nelore 3/8 Charolês e 5/8 Charolês 3/8 Nelore, Pacheco et al. (2005a) comentaram que, para o frigorífico, seria mais vantajoso o abate de animais mais pesados, conseqüentemente, com maior peso de couro (e não necessariamente com maior idade) e/ou o abate de genótipos com maior predominância de sangue zebuíno.

Quando expressos em percentagem do P e do $\mathrm{PCV}$, o componente cabeça apresentou decréscimo, enquanto o couro se manteve inalterado com o aumento no peso de abate dos animais, demonstrando que estes componentes não acompanham proporcionalmente o crescimento do P e PCV. Em seu estudo, Galvão et al. (1991) observaram decréscimo no peso do componente couro à medida que aumentou o grau de maturidade dos novilhos, passando de $11,43 \mathrm{~kg} / 100 \mathrm{~kg}$ de peso de corpo vazio nos animais abatidos com $90 \%$ do 
Tabela 2 - Médias para dias em confinamento, peso vivo inicial (PI), ganho de peso médio diário (GMD), consumo médio diário de matéria seca por animal (CMSD), por $100 \mathrm{~kg}$ de peso vivo (CMSPV) e por tamanho metabólico (CMSTM) e consumo de energia líquida de mantença (ELm), de acordo com o peso de abate

Table 2 - Means for days on feed, initial weight (IW), average daily weight gain (ADG), average daily dry matter intake per animal(DMI), per $100 \mathrm{~kg}$ of live weight(DMILW) and per metabolic weight (DMIMW) and net energy for maintenance intake (NEm), according to slaughter weight

\begin{tabular}{|c|c|c|c|c|}
\hline \multirow[t]{2}{*}{$\begin{array}{l}\text { Variável } \\
\text { Variable }\end{array}$} & \multicolumn{3}{|c|}{$\begin{array}{c}\text { Peso de abate }(\mathrm{P}) \\
\text { Slaughter weight }(\mathrm{SW})\end{array}$} & \multirow[t]{2}{*}{$\begin{array}{l}\text { Média ou equação de regressão } \\
\text { Mean or regression equation }\end{array}$} \\
\hline & 425 & 467 & 510 & \\
\hline Dias em confinamento (Days on feed) & 30 & 65 & 94 & - \\
\hline PI, $\mathrm{kg}(I W, k g)$ & 362 & 358 & 363 & $\bar{Y}=361 \pm 38,58$ \\
\hline $\mathrm{GMD}, \mathrm{kg}(A D G, \mathrm{~kg})$ & 2,10 & 1,68 & 1,56 & $\hat{\mathrm{Y}}=4,939-0,007 \mathrm{P}^{\mathrm{a}}$ \\
\hline CMSD, $\mathrm{kg}(D M I, k g)$ & 9,97 & 9,93 & 10,61 & $\bar{Y}=10,17 \pm 0,66$ \\
\hline CMSPV, $\mathrm{kg}(D M I L W, \mathrm{~kg})$ & 2,53 & 2,41 & 2,43 & $\bar{Y}=2,46 \pm 0,15$ \\
\hline CMSTM, g (DMIMW, g) & 112,79 & 108,50 & 111,02 & $\bar{Y}=110,77 \pm 6,24$ \\
\hline $\operatorname{ELm}, \operatorname{Mcal}(N E m, M c a l)$ & 6,81 & 7,05 & 7,35 & $\hat{\mathrm{Y}}=3,939+0,007 \mathrm{P}^{b}$ \\
\hline
\end{tabular}

a $R^{2}=0,81, P=0,0198 ; \quad b R^{2}=0,7012, P=0,0048$

Fonte (Source): Arboitte et al. (2004 b).

Tabela 3 - Médias para rendimentos de carcaça quente (RCQ) e fria (RCF) relativos ao peso de abate (P) e ao peso de corpo vazio (PCV), de acordo com o peso de abate

Table 3 - Means for hot (HDP) and cold (CDP) dressing percentages relative to slaughter weight (SW) and relative to empty body weight (EBW), according to slaughter weight

\begin{tabular}{|c|c|c|c|c|}
\hline \multirow[t]{2}{*}{$\begin{array}{l}\text { Variável } \\
\text { Variable }\end{array}$} & \multicolumn{3}{|c|}{$\begin{array}{l}\text { Peso de abate }(\mathrm{P}) \\
\text { Slaughter weight }(S W)\end{array}$} & \multirow[t]{2}{*}{$\begin{array}{l}\text { Média ou equação de regressão } \\
\text { Mean or regression equation }\end{array}$} \\
\hline & 425 & 467 & 510 & \\
\hline $\begin{array}{l}\text { Peso de corpo vazio (PCV), } \mathrm{kg} \\
\text { Empty body weight }(E B W), \mathrm{kg}\end{array}$ & 368 & 405 & 461 & \\
\hline $\begin{array}{l}\text { RCQ-P, \% } \\
H D P-S W, \%\end{array}$ & 54,55 & 56,64 & 56,63 & $\hat{\mathrm{Y}}=40,511( \pm 6,925)+0,010( \pm 0,011) \mathrm{Pi}^{*}+0,026( \pm 0,012) \mathrm{P}^{\mathrm{a}}$ \\
\hline $\begin{array}{l}\text { RCQ-PCV, \% } \\
H D P-E B W, \%\end{array}$ & 62,84 & 63,59 & 62,68 & $\overline{\mathrm{Y}}=63,04( \pm 1,29)$ \\
\hline $\begin{array}{l}\text { RCF-P, } \% \\
C D P-S W, \%\end{array}$ & 53,14 & 55,49 & 55,42 & 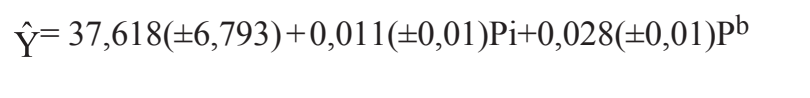 \\
\hline $\begin{array}{l}\text { RCF-PCV, \% } \\
C D P-E B W, \%\end{array}$ & 61,37 & 62,12 & 61,34 & $\bar{Y}=61,67( \pm 1,27)$ \\
\hline
\end{tabular}

${ }^{*} \mathrm{Pi}=$ peso inicial (initial weight).

${ }^{a} R^{2}=0,9100, P=0,0001 ;{ }^{b} R^{2}=0,3000, P=0,0660$.

peso adulto para $10,64 \mathrm{~kg} / 100 \mathrm{~kg}$ de peso de corpo vazio nos novilhos abatidos com $110 \%$ do peso adulto. No estudo de Jorge \& Fontes (2001), que avaliaram o desenvolvimento relativo das partes do corpo de novilhos zebuínos de quatro raças a partir dos coeficientes de alometria, cabeça, pés e couro apresentaram coeficientes menores que um, ou seja, as intensidades de desenvolvimento dessas partes do corpo foram inferiores à do peso corporal vazio, refletindo a maturidade mais precoce destes componentes.
Ainda na Tabela 4, analisando o peso absoluto total dos componentes externos, verifica-se aumento de $144 \mathrm{~g} / \mathrm{kg}$ no $\mathrm{P}$, conforme estimativa da equação de regressão, com o maior ímpeto de crescimento verificado dos 425 aos $467 \mathrm{~kg}$ (10,7\%), reduzindo para $4,7 \%$ dos 467 aos $510 \mathrm{~kg}$. No entanto, quando ajustado para $\mathrm{P}$ e PCV, houve similaridade para esta característica com o aumento no peso de abate.

As médias expressas em valores absolutos e relativos a $100 \mathrm{~kg}$ de peso de abate e de corpo vazio 
Tabela 4 - Médias para pesos expressos em valores absolutos e em percentagem do peso de abate $(P)$ e de corpo vazio (PCV) dos componentes externos, de acordo com o peso de abate

Table 4 - Means for weights expressed in absolute values and in percentage of slaughter weight (SW) and of empty body weight (EBW) of external components, according to slaughter weight

\begin{tabular}{lccc}
\hline Variável & \multicolumn{2}{c}{$\begin{array}{c}\text { Peso de abate }(\mathrm{P}) \\
\text { Slaughter weight }(S W)\end{array}$} & $\begin{array}{c}\text { Média ou equação de regressão } \\
\text { Variable }\end{array}$ \\
\cline { 2 - 3 } & 425 & 467 & 510
\end{tabular}

Componente externo

External components

\begin{tabular}{lrrrl} 
Cabeça, kg (Head, kg) & 14,42 & 14,63 & 15,11 & $\overline{\mathrm{Y}}=14,72( \pm 0,98)$ \\
Cabeça, \% P (Head, \% P) & 3,39 & 3,13 & 2,98 & $\hat{\mathrm{Y}}=6,142( \pm 0,865)-0,0017( \pm 0,001) \mathrm{Pi}^{*}-0,0052( \pm 0,002) \mathrm{P}^{\mathrm{a}}$ \\
Cabeça, \% PCV (Head, \% EBW) & 3,92 & 3,62 & 3,30 & $\hat{\mathrm{Y}}=8,043( \pm 1,117)-0,0024( \pm 0,002) \mathrm{Pi}-0,0078( \pm 0,002) \mathrm{P}^{\mathrm{b}}$ \\
Patas, kg (Feet, kg) & 7,68 & 7,89 & 8,82 & $\hat{\mathrm{Y}}=-7,68( \pm 1,736)+0,026( \pm 0,003) \mathrm{Pi}+0,014( \pm 0,003) \mathrm{P}^{\mathrm{c}}$ \\
Patas, \% P (Feet, \% P) & 1,80 & 1,68 & 1,73 & $\overline{\mathrm{Y}}=1,74( \pm 0,091)$ \\
Patas, \% PCV (Feet, \% EBW) & 2,08 & 1,94 & 1,91 & $\hat{\mathrm{Y}}=2,541( \pm 0,453)+0,0012( \pm 0,0007) \mathrm{Pi}-0,002( \pm 0,0008) \mathrm{P}^{\mathrm{d}}$ \\
Couro, kg (Rawhide, kg) & 35,15 & 43,05 & 44,68 & $\hat{\mathrm{Y}}=-52,609( \pm 18,676)+0,108( \pm 0,029) \mathrm{Pi}+0,119( \pm 0,033) \mathrm{P}^{\mathrm{e}}$ \\
Couro, \% P (Rawhide, \% P) & 8,26 & 9,25 & 8,73 & $\overline{\mathrm{Y}}=8,75( \pm 1,017)$ \\
Couro, \% PCV (Rawhide, \% EBW) & 9,55 & 10,66 & 9,66 & $\overline{\mathrm{Y}}=9,96( \pm 1,141)$ \\
Total, kg (Total, kg) & 60,31 & 66,79 & 69,94 & $\hat{\mathrm{Y}}=-60,893( \pm 18,656)+0,1646( \pm 0,029) \mathrm{Pi}+0,144( \pm 0,033) \mathrm{P}^{\mathrm{f}}$ \\
Total, \% P (Total, \% P) & 16,36 & 16,07 & 15,14 & $\overline{\mathrm{Y}}=15,86( \pm 1,036)$ \\
Total, \% PCV (Total, \% EBW) & 16,36 & 16,07 & 15,14 & $\ddot{\mathrm{Y}}=15,83( \pm 1,214)$ \\
\hline
\end{tabular}

${ }^{*} \mathrm{Pi}=$ peso inicial (initial weight).

a $R^{2}=0,4567, P=0,0046 ; \quad$ b $R^{2}=0,5307, P=0,0015 ; \quad$ c $R^{2}=0,8813, P=0,0004 ; \quad d R^{2}=0,3858, P=0,0202 ; \quad$ e $R^{2}=0,6369, P=0,0030$; ${ }^{f} R^{2}=0,7715, P=0,0006$.

para sangue e órgãos vitais, de acordo com os diferentes estádios de maturidade, constam na Tabela 5.

Quanto ao componente sangue, houve incremento linear, quando expresso em valores absolutos, e similaridade, quando ajustado para peso de abate e de corpo vazio, com o aumento no peso de abate. Em valores absolutos, estes resultados estão de acordo com os relatados por Jesse et al. (1976), que, ao abaterem novilhos Hereford com 227; 341; 454 e $545 \mathrm{~kg}$, constataram quantidade de sangue de 6,64; 9,$28 ; 12,64$ e $13,28 \mathrm{~kg}$, respectivamente.

Alguns estudos demonstraram que a variação no peso de sangue acompanha a variação no peso de órgãos vitais e de trato digestivo vazio (Ribeiro et al., 2001; Pacheco et al., 2005a), o que seria necessário para acompanhar as variações na taxa metabólica nos animais. Neste ensaio, o peso de sangue correlacionou-se positivamente com total de órgãos vitais $(\mathrm{r}=0,40 ; \mathrm{P}=0,1013)$, com total do trato digestivo vazio $(\mathrm{r}=0,32 ; \mathrm{P}=0,1974)$ e com total de gorduras internas $(\mathrm{r}=0,44 ; \mathrm{P}=0,0673)$.

Em valores absolutos, fígado apresentou aumento com o avanço no estádio de maturidade dos animais. Enquanto que os pesos dos órgãos internos, coração, pulmão e rins decresceram frente ao aumento no peso de abate quando ajustados para PCV. Segundo Berg \& Butterfield (1976), órgãos internos e vísceras apresentam desenvolvimento precoce e o fenômeno ocorre com maior intensidade nos estádios iniciais da vida do animal. No entanto, avaliando os coeficientes de alometria de diversos componentes não-integrantes da carcaça de novilhos zebuínos, Jorge \& Fontes (2001) verificaram que fígado e baço apresentaram intensidade de crescimento igual ou superior à do peso de corpo vazio.

O conjunto dos órgãos internos apresentou aumento de $17 \mathrm{~g} / \mathrm{kg}$ de aumento no peso dos animais, de acordo com estimativa da equação de regressão, sendo o fígado o principal órgão participante deste acréscimo (10 g/kg de aumento de peso). Contudo, quando foram expressos em relação a $100 \mathrm{~kg}$ do P e $\mathrm{PCV}$, o comportamento dos dados inverteu-se, com equações de regressão estimando decréscimos frente ao aumento no peso de abate. Em sua revisão, Cumby (2000) relatou que o tamanho e a taxa metabólica dos órgãos são diferentes em relação às demais partes do corpo do animal, podendo estar diretamente relacionados ao consumo de alimentos e às exigências energéticas, principalmente de energia líquida de 
Tabela 5 - Médias para pesos expressos em valores absolutos e em percentagem do peso de abate $(P)$ e de corpo vazio (PCV) de sangue e órgãos vitais, de acordo com o peso de abate

Table 5 - Means for weights expressed in absolute values and in percentage of slaughter weight (SW) and of empty body weight (EBW) of blood and of vital organs, according to slaughter weight

\begin{tabular}{|c|c|c|c|c|}
\hline \multirow[t]{2}{*}{$\begin{array}{l}\text { Variável } \\
\text { Variable }\end{array}$} & \multicolumn{3}{|c|}{$\begin{array}{c}\text { Peso de abate }(\mathrm{P}) \\
\text { Slaughter weight }(S W)\end{array}$} & \multirow[t]{2}{*}{$\begin{array}{l}\text { Média ou equação de regressão } \\
\text { Mean or regression equation }\end{array}$} \\
\hline & 425 & 467 & 510 & \\
\hline Sangue, $\mathrm{kg}$ (Blood, $\mathrm{kg}$ ) & 10,18 & 11,43 & 12,92 & $\hat{\mathrm{Y}}=-10,436( \pm 8,383)+0,017( \pm 0,013) \mathrm{Pi}^{*}+0,034( \pm 0,015) \mathrm{P}^{\mathrm{a}}$ \\
\hline Sangue, \% P (Blood, \% P) & 2,41 & 2,46 & 2,53 & $\overline{\mathrm{Y}}=2,46( \pm 0,43)$ \\
\hline Sangue, \% PCV (Blood, \% EBW) & 2,78 & 2,85 & 2,80 & $\overline{\mathrm{Y}}=2,81( \pm 0,52)$ \\
\hline \multicolumn{5}{|l|}{ Örgãos vitais (Vital organs) } \\
\hline Coração, kg (Heart, kg) & 1,49 & 1,47 & 1,49 & $\bar{Y}=1,48( \pm 0,29)$ \\
\hline Coração, \% P (Heart, \% P) & 0,35 & 0,31 & 0,29 & $\hat{\mathrm{Y}}=0,737( \pm 0,224)-0,0002( \pm 0,0004) \mathrm{Pi}-0,0007( \pm 0,0004) \mathrm{P}^{\mathrm{b}}$ \\
\hline Coração, \% PCV (Heart, \% EBW) & 0,40 & 0,37 & 0,32 & $\hat{\mathrm{Y}}=0,949( \pm 0,285)-0,0003( \pm 0,0004) \mathrm{Pi}-0,001( \pm 0,0005) \mathrm{P}^{\mathrm{c}}$ \\
\hline Pulmão, kg (Lung, kg) & 5,30 & 5,02 & 5,75 & $\ddot{Y}=5,36( \pm 0,57)$ \\
\hline Pulmão, \% P (Lung, \% P) & 1,24 & 1,08 & 1,13 & $\ddot{Y}=1,15( \pm 0,12)$ \\
\hline Pulmão, \% PCV (Lung, \%EBW) & 1,44 & 1,24 & 1,25 & $\hat{\mathrm{Y}}=2,654( \pm 0,05)-0,0008( \pm 0,0008) \mathrm{Pi}-0,002( \pm 0,0009) \mathrm{P}^{\mathrm{d}}$ \\
\hline Rins, $\mathrm{kg}$ (Kidney, kg) & 0,75 & 0,77 & 0,82 & $\overline{\mathrm{Y}}=0,78( \pm 0,08)$ \\
\hline Rins, \% P (Kidney, \% P) & 0,18 & 0,16 & 0,16 & $\bar{Y}=0,17( \pm 0,02)$ \\
\hline Rins, \% PCV (Kidney, \%EBW) & 0,20 & 0,19 & 0,18 & $\hat{\mathrm{Y}}=, 393( \pm 0,093)-0,0001( \pm 0,0002) \mathrm{Pi}-0,0004( \pm 0,0002) \mathrm{P}^{\mathrm{e}}$ \\
\hline Fígado, $\mathrm{kg}$ (Liver, $k g$ ) & 4,88 & 5,13 & 5,66 & $\hat{\mathrm{Y}}=-1,543( \pm 2,030)+0,006( \pm 0,003) \mathrm{Pi}+0,010( \pm 0,004) \mathrm{P}^{\mathrm{f}}$ \\
\hline Fígado, \% P (Liver, \% P) & 1,16 & 1,10 & 1,11 & $\bar{Y}=1,12( \pm 0,11)$ \\
\hline Fígado, \% PCV (Liver, \% EBW) & 1,34 & 1,28 & 1,23 & $\overline{\mathrm{Y}}=1,28( \pm 0,15)$ \\
\hline Baço, kg (Spleen, kg) & 1,40 & 1,35 & 1,45 & $\ddot{Y}=1,40( \pm 0,33)$ \\
\hline Baço, \% P (Spleen,\% P) & 0,34 & 0,28 & 0,29 & $\overline{\bar{Y}}=0,30( \pm 0,08)$ \\
\hline Baço, \% PCV (Spleen, \% EBW) & 0,39 & 0,33 & 0,32 & $\overline{\mathrm{Y}}=0,34( \pm 0,09)$ \\
\hline Total, $\mathrm{kg}$ (Total, $\mathrm{kg})$ & 13,81 & 13,74 & 15,16 & $\hat{\mathrm{Y}}=-2,260( \pm 4,814)+0,024( \pm 0,008) \mathrm{Pi}+0,017( \pm 0,008) \mathrm{P}^{\mathrm{g}}$ \\
\hline Total, \% P (Total, \% P) & 3,26 & 2,95 & 2,98 & $\hat{\mathrm{Y}}=5,797( \pm 1,039)-0,003( \pm 0,002) \mathrm{Pi}-0,004( \pm 0,002) \mathrm{P}^{\mathrm{h}}$ \\
\hline Total, \% PCV (Total, \% EBW) & 3,77 & 3,41 & 3,30 & $\hat{\mathrm{Y}}=7,625( \pm 1,249)-0,004( \pm 0,002) \mathrm{Pi}-0,006( \pm 0,002) \mathrm{P}^{\mathrm{i}}$ \\
\hline
\end{tabular}

${ }^{*} \mathrm{Pi}=$ peso inicial (initial weight).

a $R^{2}=0,3154, P=0,0387 ; \quad b R^{2}=0,2001, P=0,0870 ; c R^{2}=0,2318, P=0,0634 ; \quad d R^{2}=0,3363, P=0,0225 ; \quad$ e $R^{2}=0,2466, P=0,0614 ;$ ${ }^{f} R^{2}=0,4265, P=0,0175 ;{ }^{9} R^{2}=0,4871, P=0,0709 ;{ }^{h} R^{2}=0,3237, P=0,0769 ;{ }^{i} R^{2}=0,4233, P=0,0193$.

mantença. Dos órgãos vitais, Owens et al. (1993) e Ferrel \& Jenkins (1998) citaram que o fígado é o mais alterado frente às variações no consumo de alimentos, exigências energéticas e taxas metabólicas, pois participa ativamente no metabolismo dos nutrientes. Observou-se que o consumo de matéria seca (em kg, em percentagem do peso vivo e por unidade de tamanho metabólico) não foi alterado, o ganho de peso médio diário $(\mathrm{kg})$ reduziu linearmente e a exigência de energia líquida de mantença (ELm), em Mcal, elevou linearmente com o aumento no peso de abate dos animais. De acordo com análise de correlação entre estas três características e o peso de fígado $(\mathrm{kg})$, os coeficientes obtidos foram de $0,91(\mathrm{P}=0,0128),-0,07$ $(\mathrm{P}=0,7915)$ e $0,91(\mathrm{P}=0,0122)$, respectivamente.
Pacheco et al. (2005a) verificaram que a correlação entre peso de fígado (em valores absolutos) e exigência de energia líquida de mantença (Mcal) foi de 0,57.

Os pesos absolutos e relativos a $100 \mathrm{~kg}$ de $\mathrm{P}$ e $\mathrm{PCV}$ das diferentes gorduras internas, de acordo com o peso de abate encontram-se na Tabela 6 .

Os pesos de gordura ruminal e inguinal incrementaram linearmente frente ao aumento no peso de abate dos animais, tanto em valores absolutos quanto em percentagem do peso de abate e de corpo vazio, de modo que a primeira apresentou maior acréscimo ( $97 \mathrm{~g} / \mathrm{kg}$ de aumento no peso de abate), conforme estimativa da equação de regressão. Segundo análise dos coeficientes de alometria estimados no estudo de Restle et al. (2005), que trabalharam com 
Tabela 6 - Médias para pesos expressos em valores absolutos e em percentagem do peso de abate $(P)$ e de corpo vazio (PCV) das gorduras internas, de acordo com o peso de abate

Table 6 - Means for weights expressed in absolute values and in percentage of slaughter weight (SW) and of empty body weight (EBW) of internal fats, according to slaughter weight

\begin{tabular}{|c|c|c|}
\hline \multirow{3}{*}{$\begin{array}{l}\text { Variável } \\
\text { Variable }\end{array}$} & Peso de abate (P) & \multirow{3}{*}{$\begin{array}{c}\text { Média ou equação de regressão } \\
\text { Mean or regression equation }\end{array}$} \\
\hline & Slaughter weight (SW) & \\
\hline & 467 & \\
\hline
\end{tabular}

\section{Gordura interna}

Internal fat

\begin{tabular}{lrrrl} 
Ruminal, kg (Ruminal, kg) & 6,28 & 5,92 & 14,05 & $\hat{\mathrm{Y}}=-39,878( \pm 11,477)+0,011( \pm 0,018) \mathrm{Pi}^{*}+0,097( \pm 0,02) \mathrm{P}^{\mathrm{a}}$ \\
Ruminal, \% P (Ruminal, \% P) & 1,47 & 1,28 & 2,77 & $\hat{\mathrm{Y}}=-5,078( \pm 2,456)-0,002( \pm 0,004) \mathrm{Pi}+0,016( \pm 0,004) \mathrm{P}^{\mathrm{b}}$ \\
Ruminal, \% PCV (Ruminal, \%EBW) & 1,69 & 1,48 & 3,07 & $\hat{\mathrm{Y}}=-5,160( \pm 2,723)-0,002( \pm 0,004) \mathrm{Pi}+0,017( \pm 0,005) \mathrm{P}^{\mathrm{c}}$ \\
Inguinal, kg (Inguinal, kg) & 1,94 & 3,35 & 4,92 & $\hat{\mathrm{Y}}=-15,477( \pm 3,529)+0,005( \pm 0,006) \mathrm{Pi}+0,037( \pm 0,006) \mathrm{P}^{\mathrm{d}}$ \\
Inguinal, \% P (Inguinal, \% P) & 0,45 & 0,73 & 0,96 & $\hat{\mathrm{Y}}=-1,964( \pm 0,666)-0,0006( \pm 0,001) \mathrm{Pi}+0,006( \pm 0,001) \mathrm{P}^{\mathrm{e}}$ \\
Inguinal, \% PCV (Inguinal, \% EBW) & 0,52 & 0,83 & 1,06 & $\hat{\mathrm{Y}}=-2,024( \pm 0,726)-0,0007( \pm 0,001) \mathrm{Pi}+0,007( \pm 0,001) \mathrm{P}^{\mathrm{f}}$ \\
Renal, kg (Kidney, kg) & 3,65 & 5,22 & 6,18 & $\hat{\mathrm{Y}}=-14,779( \pm 6,716)+0,015( \pm 0,005) \mathrm{P}+0,032( \pm 0,012) \mathrm{P}^{\mathrm{g}}$ \\
Renal, \% P (Kidney, \% P) & 0,86 & 1,13 & 1,19 & $\hat{\mathrm{Y}}=1,06( \pm 0,35)$ \\
Renal, \% PCV (Kidney, \% EBW) & 0,99 & 1,31 & 1,31 & $\overline{\mathrm{Y}}=1,20( \pm 0,39)$ \\
Toalete, kg (Trim, kg) & 1,85 & 3,11 & 2,79 & $\hat{\mathrm{Y}}=-5,012( \pm 3,479)+0,006( \pm 0,005) \mathrm{P}+0,012( \pm 0,006) \mathrm{P}^{\mathrm{h}}$ \\
Toalete, \% P (Trim, \% P) & 0,43 & 0,67 & 0,54 & $\hat{\mathrm{Y}}=0,55( \pm 0,19)$ \\
Toalete, \% PCV (Trim, \% EBW) & 0,49 & 0,77 & 0,60 & $\hat{\mathrm{Y}}=0,62( \pm 0,20)$ \\
Total, kg (Total, kg) & 13,72 & 17,60 & 27,93 & $\hat{\mathrm{Y}}=-5,146( \pm 15,265)+0,037( \pm 0,024) \mathrm{Pi}+0,177( \pm 0,027) \mathrm{P}^{\mathrm{i}}$ \\
Total, \% P (Total, \% P) & 3,21 & 3,81 & 5,46 & $\hat{\mathrm{Y}}=-8,321( \pm 3,02)-0,001( \pm 0,005) \mathrm{Pi}+0,029( \pm 0,005) \mathrm{P}^{\mathrm{j}}$ \\
Total, \% PCV (Total, \% EBW) & 3,70 & 4,39 & 6,05 & $\hat{\mathrm{Y}}=-8,164( \pm 3,228)-0,002( \pm 0,005) \mathrm{Pi}+0,029( \pm 0,006) \mathrm{P}^{\mathrm{k}}$ \\
\hline
\end{tabular}

* $\mathrm{Pi}=$ peso inicial (initial weight).

a $R^{2}=0,6010, P=0,0003 ; \quad b \quad R^{2}=0,4810, P=0,0021 ; c R^{2}=0,4582, P=0,0030 ; d R^{2}=0,7018, P=0,0001 ; \quad$ e $R^{2}=0,6439, P=0,0001 ;$

f $R^{2}=0,6527, P=0,0001 ; g \quad R^{2}=0,3703, P=0,0194 ; h \quad R^{2}=0,2422, P=0,0810 ; \quad R^{2}=0,7475, P=0,0001 ; j \quad R^{2}=0,6470, P=0,0001$;

${ }^{k} R^{2}=0,6332, P=0,0001$.

os mesmos animais utilizados neste estudo, verificouse que, quando relacionado com o aumento no peso de corpo vazio, as gorduras inguinal e ruminal apresentaram coeficientes de 3,67 e 3,27, respectivamente, superiores aos coeficientes das gorduras de toalete $(2,29) \mathrm{e}$ renal $(1,80)$, indicando que as gorduras internas apresentam crescimento mais acentuado em relação ao aumento no peso de corpo vazio, ou seja, maturidade mais tardia. Apesar de serem poucos os estudos que quantificaram coeficientes de alometria dos componentes do corpo vazio em relação ao peso de corpo vazio, vários resultados de trabalhos envolvendo terminação de novilhos demonstraram que existe alteração na composição do ganho de peso com o avanço no período de alimentação, passando a depositar mais tecido adiposo que muscular (Berg \& Butterfield, 1976; Restle et al., 1997 b; Bail et al., 2000; Restle et al., 2000; Costa et al., 2002b; Restle et al., 2003; Arboitte et al., 2004b; Pacheco et al., 2005b).
Com o aumento no peso de abate, houve maior porcentagem de gordura de toalete, quando expresso em valores absolutos, e similaridade, quando ajustados para peso de abate e de corpo vazio. Verifica-se que, para todos os pesos de abate, houve penalização das carcaças com o recorte do excesso de gordura, mesmo para aquelas dos animais abatidos com $425 \mathrm{~kg}$, que apresentaram espessura de gordura subcutânea dentro dos limites exigidos pelos frigoríficos (Arboitte et al., 2004a), o que representa perdas econômicas ao produtor, posto que o acúmulo de gordura é um processo menos eficiente que o acúmulo dos demais tecidos que compõem a carcaça, resultando em maior gasto com alimentação. Para o frigorífico, este tipo de gordura também representaria prejuízo econômico se estivesse incluída no peso de carcaça, uma vez que após resfriamento, seria comercializada separadamente por preço inferior, embutindo-se, ainda, todos os demais custos de produção. Somente não teriam 
Tabela 7 - Médias para pesos expressos em valores absolutos e em percentagem do peso de abate $(P)$ e de corpo vazio (PCV) do trato gastrintestinal vazio, de acordo com o peso de abate

Table 7 - Means for weights expressed in absolute values and in percentage of slaughter weight (SW) and of empty body weight (EBW) of empty gastrintestinal tract, according to slaughter weight

\begin{tabular}{lccc}
\hline Variável & \multicolumn{2}{c}{$\begin{array}{c}\text { Peso de abate }(\mathrm{P}) \\
\text { Slaughter weight }(\mathrm{SW})\end{array}$} & $\begin{array}{c}\text { Média ou equação de regressão } \\
\text { Variable }\end{array}$ \\
\cline { 2 - 3 } & 425 & 467 & 510
\end{tabular}

Trato gastrintestinal vazio

Empty gastrintestinal tract

\begin{tabular}{|c|c|c|c|c|}
\hline $\begin{array}{l}\text { Rúmen-retículo, } \mathrm{kg} \\
\text { Rumen-reticulum, kg }\end{array}$ & 9,27 & 8,85 & 10,02 & $\overline{\mathrm{Y}}=9,37( \pm 1,45)$ \\
\hline $\begin{array}{l}\text { Rúmen-retículo, \% } \mathrm{P} \\
\text { Rumen-reticulum, \% P }\end{array}$ & 2,18 & 1,90 & 1,97 & $\overline{\mathrm{Y}}=2,02( \pm 0,28)$ \\
\hline $\begin{array}{l}\text { Rúmen-retículo, \% PCV } \\
\text { Rumen-reticulum, \% EBW }\end{array}$ & 2,52 & 2,20 & 2,18 & $\hat{\mathrm{Y}}=-7,196+0,009 \mathrm{Pi}^{*}+0,017 \mathrm{P}^{\mathrm{a}}$ \\
\hline $\begin{array}{l}\text { Omaso, } \mathrm{kg} \\
\text { Omasum, kg }\end{array}$ & 1,58 & 1,63 & 1,78 & $\overline{\mathrm{Y}}=1,67( \pm 0,36)$ \\
\hline $\begin{array}{l}\text { Omaso, \% } \mathrm{P} \\
\text { Omasum, } \% \text { P }\end{array}$ & 0,37 & 0,35 & 0,35 & $\bar{Y}=0,36( \pm 0,07)$ \\
\hline $\begin{array}{l}\text { Omaso, \% PCV } \\
\text { Omasum, \% EBW }\end{array}$ & 0,43 & 0,41 & 0,39 & $\overline{\mathrm{Y}}=0,41( \pm 0,08)$ \\
\hline $\begin{array}{l}\text { Abomaso, } \mathrm{kg} \\
\text { Abomasum, } \mathrm{kg}\end{array}$ & 3,38 & 3,68 & 4,73 & $\hat{\mathrm{Y}}=-7,196( \pm 2,945)+0,009( \pm 0,005) \mathrm{Pi}+0,017( \pm 0,005) \mathrm{P}^{\mathrm{b}}$ \\
\hline $\begin{array}{l}\text { Abomaso, } \% \mathrm{P} \\
\text { Abomasum, \% P }\end{array}$ & 0,79 & 0,78 & 0,94 & $\overline{\mathrm{Y}}=0,84( \pm 0,16)$ \\
\hline $\begin{array}{l}\text { Abomaso, \% PCV } \\
\text { Abomasum, \% EBW }\end{array}$ & 0,92 & 0,90 & 1,04 & $\bar{Y}=0,95( \pm 0,18)$ \\
\hline $\begin{array}{l}\text { IDG**, } \mathrm{kg} \\
S L I^{* *}, \mathrm{~kg}\end{array}$ & 11,45 & 12,80 & 14,33 & $\hat{\mathrm{Y}}=-2,468( \pm 9,521)+0,024( \pm 0,015) \mathrm{Pi}+0,036( \pm 0,017) \mathrm{P}^{\mathrm{c}}$ \\
\hline $\begin{array}{l}\mathrm{IDG}^{* *} \% \mathrm{P} \\
S L I^{* *}, \% P\end{array}$ & 2,72 & 2,74 & 2,83 & $\bar{Y}=2,76( \pm 0,54)$ \\
\hline $\begin{array}{l}\mathrm{IDG}^{* *}, \% \mathrm{PCV} \\
S L I^{* *}, \% E B W\end{array}$ & 3,14 & 3,16 & 3,13 & $\bar{Y}=3,14( \pm 0,62)$ \\
\hline $\begin{array}{l}\text { Total, } \mathrm{kg} \\
\text { Total, } \mathrm{kg}\end{array}$ & 25,68 & 26,97 & 30,87 & $\hat{\mathrm{Y}}=-20,696( \pm 10,405)+0,052( \pm 0,016) \mathrm{Pi}+0,065( \pm 0,019) \mathrm{P}^{\mathrm{d}}$ \\
\hline $\begin{array}{l}\text { Total, \% } \mathrm{P} \\
\text { Total, \% P }\end{array}$ & 6,06 & 5,77 & 6,08 & $\overline{\mathrm{Y}}=5,97( \pm 0,57)$ \\
\hline $\begin{array}{l}\text { Total, \% PCV } \\
\text { Total, \% } E B W\end{array}$ & 7,00 & 6,66 & 6,73 & $\overline{\mathrm{Y}}=6,80( \pm 0,67)$ \\
\hline
\end{tabular}

${ }^{*} \mathrm{Pi}=$ peso inicial (initial weight)

** Intestinos delgado + grosso (small plus large intestines).

a $R^{2}=0,2561, P=0,0986 ;{ }^{b} R^{2}=0,4887, P=0,0062 ;{ }^{c} R^{2}=0,3222, P=0,0527 ;{ }^{d} R^{2}=0,5981, P=0,0035$.

prejuízos se existissem mercados com demanda por carcaças com maior quantidade de gordura, como por exemplo, países asiáticos.

Observou-se avanço linear no peso total das gorduras internas com o aumento do peso de abate, independentemente da forma de expressão. De acordo com estimativa da equação de regressão, cada $\mathrm{kg}$ de aumento do peso de abate representou incremento de $177 \mathrm{~g}$ no peso total de gorduras internas. Para o produtor, a importância deste resultado se deve à relação positiva entre exigências energéticas e peso das gorduras internas. Conforme NRC (1996), o teor de gordura interna e o peso adulto são características importantes na estimativa das exigências energéticas dos animais, principalmente a ELm. Gesualdi Jr. et al. (2001) acrescentam que incrementos nos requerimentos para mantença de animais com excesso de gorduras internas, também ocorre pelo fato de a 
atividade metabólica destes tecidos ser maior que nos tecidos periféricos. Neste estudo, a correlação entre gorduras internas e ELm foi de 0,78 ( $\mathrm{P}=0,0660)$.

$\mathrm{Na}$ Tabela 7 , estão apresentados os resultados referentes aos pesos absolutos e relativos a $100 \mathrm{~kg}$ de peso de abate e de corpo vazio dos componentes do trato gastrintestinal vazio, de acordo com o peso de abate dos animais.

Com exceção dos pesos absolutos de abomaso, intestinos delgado + grosso, total do trato gastrintestinal vazio e do peso ajustado para PCV de rúmen + retículo, que apresentaram incremento, os demais componentes expressos nas diferentes maneiras não diferiram com o avanço no peso de abate dos animais. No estudo de Galvão et al. (1991), não se verificaram diferenças no peso relativo do trato gastrintestinal + mesentério e no comprimento do intestino grosso. No entanto, houve acréscimo no comprimento do intestino delgado quando os autores avaliaram carcaças de novilhos Nelore e seus mestiços meio sangue Limousin e Marchigiana, abatidos com pesos equivalentes a 90, 100 e $110 \%$ do peso adulto. Por outro lado, Jorge et al. (1999) observaram declínio do conjunto rúmen + retículo + omaso + abomaso e intestino delgado, expressos em relação a $100 \mathrm{~kg}$ de peso de corpo vazio, quando o peso de abate elevou de 405 para $500 \mathrm{~kg}$.

Vários estudos (Signoretti et al., 1999; Véras et al., 2001; Gesualdi Jr. et al., 2001) demonstraram que a variação no peso de abomaso está relacionada principalmente ao efeito nutricional. Segundo Ferrel et al. (1976), este órgão participa ativamente do processo de digestão, podendo ser maior em animais que recebem altos níveis de concentrado. Pacheco et al. (2005a) acrescentaram que, mesmo com a mesma relação volumoso:concentrado, o peso de abomaso pode apresentar variação em razão do tipo de seleção feita por parte dos animais, ou seja, priorizando em maior ou menor grau a fração concentrado.

Em relação aos demais componentes do trato digestivo, resultados de estudos avaliando os componentes não-integrantes da carcaça de bovinos demonstraram que o peso destes componentes pode variar de acordo com consumo de alimentos, histórico nutricional, grupo genético dos animais (Hoog, 1991; Santos et al., 2003; Pacheco et al., 2005a) e tipo da dieta (volumosa ou concentrada) (Gesualdi Jr. et al., 2001; Ribeiro et al., 2001). Segundo dados de desem- penho constantes na Tabela 2 (Arboitte et al., 2004 b), o consumo de matéria seca, expresso em $\mathrm{kg}$, em percentagem do peso vivo e por unidade de tamanho metabólico, não variou com o aumento no peso de abate. A relação entre consumo de matéria seca e peso do trato digestivo vazio foi positiva $(\mathrm{r}=0,52)$, embora não-significativa $(\mathrm{P}=0,2926)$.

\section{Conclusões}

O incremento no peso de abate proporcionou maiores rendimentos de carcaças quente e fria, quando expressos por peso de abate, e similaridade, quando expressos por peso de corpo vazio.

Em valores absolutos, o peso total dos componentes externos, total de órgãos vitais, total de gorduras internas e total do trato gastrintestinal vazio elevaram com o aumento no peso de abate. No entanto, quando ajustados para peso de abate e peso de corpo vazio, os pesos totais dos componentes externos e do trato gastrintestinal vazio apresentaram similaridade, o total de órgãos vitais decresceu e o total de gorduras internas elevou com o aumento no peso de abate.

Houve relação positiva entre pesos absolutos de fígado, total de gorduras internas e total do trato gastrintestinal vazio com as características de desempenho consumo de matéria seca, em $\mathrm{kg}$, e exigência de energia líquida de mantença, em Mcal.

\section{Literatura Citada}

ARBOITTE, M.Z.; RESTlE, J.; ALVES FILHO, D.C. et al. Características da carcaça de novilhos $5 / 8$ Nelore - $3 / 8$ Charolês abatidos em diferentes estádios de desenvolvimento. Revista Brasileira de Zootecnia, v.33, n.4, p.969-977, 2004a.

ARBOITTE, M.Z.; RESTLE, J.; ALVES FILHO, D.C. et al. Desempenho em confinamento de novilhos $5 / 8$ Nelore 3/8 Charolês, abatidos em diferentes estádios de desenvolvimento. Revista Brasileira de Zootecnia, v.33, n.4, p.947-958, 2004b.

BAIL, C.A.T.; BRONDANI, I.L.; RESTLE, J. Níveis de concentrado na fase de terminação em confinamento para novilhos previamente mantidos em pastagem nativa ou cultivada. Ciência Rural, v.30, n.1, p.151-157, 2000.

BERG, R.T.; BUTTERFIELD, R.M. New concepts of cattle growth. 1.ed. Sydney: Sydney University Press, 1976. 240p.

COSTA, E.C.; RESTLE, J.; VAZ, F.N. et al. Características da carcaça de novilhos Red Angus superprecoces abatidos com diferentes pesos. Revista Brasileira de Zootecnia, v.31, n.1, p.119-128, 2002a.

COSTA, E.C.; RESTLE, J.; VAZ, F.N. et al. Desempenho de novilhos Red Angus superprecoces, confinados e abatido com diferentes pesos. Revista Brasileira de Zootecnia, v.31, n.1, p.129-138, 2002b. 
CUMBY, J. Visceral organ development during restriction and realimentation. In: CANT, J. (Ed.) Proceedings of the 2000 Course in Ruminant Digestion and Metabolism - ANSC 6260. Guelph: University of Guelph, 2000. p.23-29.

FERREL, C.L.; GARRET, W.N.; HINMAN, N. Estimation of body composition in pregnant and non-pregnant heifers. Journal of Animal Science, v.42, n.5, p.1158-1166, 1976.

FERREL, C.L.; JENKIS, T.G. Body composition and energy utilization by steers of diverse genotypes fed a high-concentrate diet during the finishing period: Angus, Boran, Brahman, Hereford and Tuli Sires. Journal of Animal Science, v.76, p.647-657, 1998.

GALVÃO, J.G.; FONTES, C.A.A.; PIRES, C.C. et al. Características e composição da carcaça de bovinos não castrados, abatidos em três estágios de maturidade (estudo II) de três grupos raciais. Revista da Sociedade Brasileira de Zootecnia, v.20, n.5, p.502-512, 1991.

GESUALDI JR., A.; VELOSO, C.M.; PAULINO, M.F. et al. Níveis de concentrado na dieta de bovinos F1 Limousin X Nelore: peso dos órgãos internos e trato digestivo. Revista Brasileira de Zootecnia, v.30, n.6, p.1866-1871, 2001.

HOOG, B.W. Compensatory growth in ruminants. In: PEARSON, A.M.; DUTSON, T.R. (Eds.). Growth regulation in farm animals. London: Elsevier Applied Science, 1991.p.103-134.

JESSE, W.G.; THOMPSON, G.B.; CLARCK, J.L. et al. Effects of ration energy and slaughter weight on composition of empty body and carcass gain of beef cattle. Journal of Animal Science, v.43, n.2, p.418-425. 1976.

JORGE, A.M.; FONTES, C.A.A. Desenvolvimento relativo das partes do corpo de zebuínos de quatro raças. Ciência Rural, v.31, n.5, p.857-861, 2001.

JORGE, A.M.; FONTES, C.A.A.; PAULINO, M.F. et al. Tamanho dos órgãos internos de zebuínos sob alimentação restrita e ad libitum. Revista Brasileira de Zootecnia, v.28, n.2, p.374-380, 1999.

NATIONAL RESEARCH COUNCIL - NRC. Nutrient requirements of domestic animals. 7.rev.ed. Washington, D.C.: National Academy Press, 1996. 242p.

OLIVEIRA, R.F.M.; FONTES, C.A.A.; CARNEIRO, L.H.D. et al. Biometria do trato gastrintestinal de bovinos de três grupos genéticos. Revista Brasileira de Zootecnia, v.21, n.2, p.205-210, 1992.

OWENS, F.N.; DUBESKI, P.; HANSON, C. F. Factors that alter the growth and development of ruminants. Journal of Animal Science, v.71, p.3138-3150, 1993.

PACHECO, P.S.; RESTLE, J.; SILVA, J.H.S. et al. Características das partes do corpo não-integrantes da carcaça de novilhos jovens e superjovens de diferentes grupos genéticos. Revista Brasileira de Zootecnia, NO PRELO, 2005a.

PACHECO, P.S.; RESTLE, J.; SILVA, J.H.S. et al. Desempenho de novilhos jovens e superjovens de diferentes grupos genéticos terminados em confinamento. Revista Brasileira de Zootecnia, v.34, n.3, p.963-975, 2005b.

RESTLE, J. Comportamento reprodutivo do rebanho de gado de corte da fazenda experimental de criação experimental agronômica da UFRGS. $1^{\circ}$ Semestre, 1972. Seminário da disciplina de Técnicas de Pesquisa. Curso de Pós-Graduação em Agronomia, Universidade Federal do Rio Grande do Sul, 1972.
RESTle, J.; ALVES FILHO, D.C.; PASCOAL, L.L. et al. Terminação em confinamento de novilhos com diferentes pesos iniciais. In: REUNIÃO ANUAL DA SOCIEDADE BRASILEIRA DE ZOOTECNIA, 40., 2003, Santa Maria. Anais... Santa Maria: SBZ [2003], CD ROM, cód 6_327.

RESTLE, J.; KEPLIN, L.A.S.; VAZ, F.N. Características quantitativas da carcaça de novilhos Charolês, abatidos com diferentes pesos. Pesquisa Agropecuária Brasileira, v.32, n.8, p.851-856, 1997a.

RESTLE, J.; KEPLIN, L.A.S.; VAZ, F.N. Desempenho em confinamento de novilhos Charolês terminados com diferentes pesos. Pesquisa Agropecuária Brasileira, v.32, n.8, p.857-860, 1997b.

RESTLE, J.; PACHECO, P.S.; ARBOITTE, M.Z. et al. Coeficientes de alometria das partes do corpo não integrantes da carcaça em relação ao peso de abate e de corpo vazio de novilhos $5 / 8$ Nelore $3 / 8$ Charolês abatidos em três estádios de desenvolvimento. Ciência Rural, NO PRELO, 2005.

RESTLE, J.; QUADROS, A.R.B.; VAZ, F.N. Terminação em confinamento de novilhos de diferentes genótipos de Hereford x Nelore. Revista Brasileira de Zootecnia, v.29, n.1, p.125-130, 2000 .

RIBEIRO, T.R.; PEREIRA, J.C.; LEÃO, M.I. et al. Tamanho dos órgãos e vísceras de bezerros holandeses, para a produção de vitelos, recebendo dietas com diferentes níveis de concentrado. Revista Brasileira de Zootecnia, v.30, n.6S, p.21632168, 2001.

SANTOS, A.P.; RESTLE, J.; PASCOAL, L.L. et al. Influência do grupo genético e da dieta alimentar no peso do corpo vazio e trato gastrintestinal de novilhos superprecoces. In: REUNIÃO ANUAL DA SOCIEDADE BRASILEIRA DE ZOOTECNIA, 40., 2003, Santa Maria, RS. Anais... Santa Maria: SBZ [2003], CD-ROM, cód 6_176.

STATISTICAL ANALYSES SYSTEM - SAS. User's guide statistics. 4 ed., Version 6, Cary: 1997. v.2, 943p.

SIGNORETTI, R.D.; ARAÚJO, G.G.L; SILVA, J.F.C. et al. Características das partes não integrantes da carcaça animal e desenvolvimento do trato gastrintestinal de bezerros da raça Holandesa alimentados com dietas contendo quatro níveis de concentrado. Revista Brasileira de Zootecnia, v.28, n.4, p.875-882, 1999.

VÉRAS, A.S.C.; VALADARES FILHO, S.C.; SILVA, J.F.C. et al. Efeito do nível de concentrado sobre o peso dos órgãos internos e do conteúdo gastrintestinal de bovinos Nelore não castrados. Revista Brasileira de Zootecnia, v.30, n.3, p.11201126, 2001 (suplemento 1). 